\title{
RDUS
}

Revue de DROIT

UNIVERSITÉ DE SHERBROOKE

Titre : $\quad$ LES CONVENTIONS MATRIMONIALES ET LE NOUVEAU DROIT

Auteur(s): $\quad$ Guy LEFRANÇOIS

Revue: $\quad$ RDUS, 1992-1993, volume 23, numéro 1

Pages: $\quad$ 203-212

ISSN : $\quad 0317-9656$

Éditeur: $\quad$ Université de Sherbrooke. Faculté de droit.

URI : $\quad$ http://hdl.handle.net/11143/13386

DOI : https://doi.org/10.17118/11143/13386 
Page vide laissée intentionnellement. 


\section{LES CONVENTIONS MATRIMONIALES ET LE NOUVEAU DROIT}

par Guy LEFRANÇOIS*

\section{SOMMAIRE}

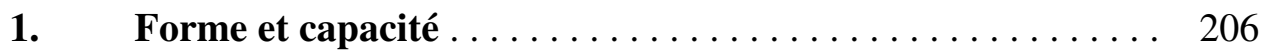

2. Contenu .............................. 207

$2.1 \quad$ Le régime matrimonial $\ldots \ldots \ldots \ldots \ldots \ldots \ldots \ldots \ldots . \ldots 208$

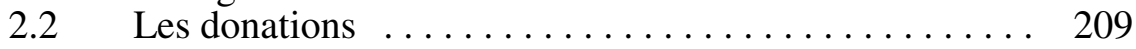

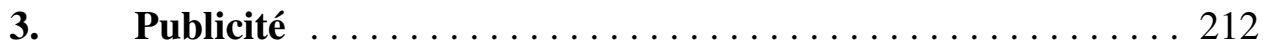

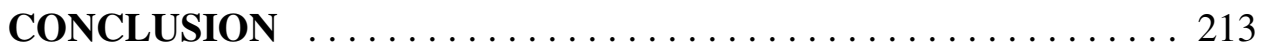

*. Notaire et professeur à la Faculté de droit de l'Université de Sherbrooke. 

et le nouveau droit

(1992) 23 R.D.U.S. 
Les statistiques en matière de conventions matrimoniales nous laissent voir que le contrat de mariage est de moins en moins utilisé au Québec, particulièrement depuis le début des années quatre-vingt ${ }^{1}$. Cette situation s'expliquerait par la baisse du nombre de mariages ${ }^{2}$ mais également, et peut-être surtout, par la transformation qu'ont subi les rapports économiques entre époux suite aux réformes de 1981 et de 1989.

La mise en place de nombreuses mesures impératives, telles que la prestation compensatoire et le patrimoine familial, a en effet remis en cause la pertinence même du contrat de mariage. Pourtant, cet instrument se justifie encore aujourd'hui. Comme certains l'ont déjà souligné ${ }^{3}$, il permet de choisir un régime matrimonial différent du régime légal, qui ne convient pas nécessairement à tous. Il peut contenir des libéralités, notamment une institution contractuelle, utile en l'absence de testament. Il peut enfin être l'occasion d'énumérer les biens possédés lors du mariage et d'en indiquer la valeur, ce qui facilite un éventuel partage du patrimoine familial ou du régime.

Les conventions matrimoniales présentent donc toujours un intérêt et dans cette perspective, il nous paraît approprié de faire le point sur l'impact qu'aura la réforme du Code civil à leur égard. C'est ce dont nous traiterons dans la présente chronique en abordant successivement trois thèmes : la forme du contrat et la capacité des parties, le contenu et la publicité.

\section{Forme et capacité}

Au sujet de la forme d'abord, précisons que le nouvel article 440 C.c.Q. reprend essentiellement le contenu de l'actuel article 472 C.c.Q. sauf pour un changement mineur dans la formulation. Par conséquent, on maintient l'exigence selon laquelle le contrat de mariage doit être fait par acte notarié portant minute, à peine de nullité absolue.

La modification des conventions matrimoniales demeure possible tant avant qu'après le mariage moyennant le respect des mêmes conditions que dans le droit actuel, c'est-à-dire la forme notariée en minute pour l'acte constatant la modification et l'obtention des consentements requis ${ }^{4}$.

1. André COSSETTE, «Statistiques en matière de mariage», (1991) 93 R. du N. 536.

2. $I d$.

3. Pierre CIOTOLA et Nicole GAGNON, «Droit actuel et nouveau droit : le point sur le patrimoine familial, la société d'acquêts et les conventions matrimoniales», (1992) 2 C.P. du N. 1, 53-56; Chambre des notaires du Québec, «Patrimoine familial et contrat de mariage», Les Cahiers, vol. 15, n 3, mars 1993, p. 698.

4. $\quad$ Art. 437 à 439 C.c.Q. 
Quant à la capacité des parties, c'est le statu quo. Le nouveau code, tout comme celui en vigueur maintenant, prévoit qu'un mineur ayant la permission de se marier peut conclure un contrat de mariage après avoir obtenu l'autorisation du tribunal donnée sur l'avis du titulaire de l'autorité parentale ou, le cas échéant, du tuteur ${ }^{5}$. Le majeur en tutelle requiert, pour sa part, l'assistance de son tuteur, dûment autorisé par le tribunal sur l'avis du conseil de tutelle, tandis que la personne pourvue d'un conseiller doit agir avec l'assistance de celui-ci ${ }^{6}$. Dans tous les cas, la violation de ces règles entraîne la nullité relative de l'acte qui ne peut toutefois être soulevée après un délai d'un an à compter de la célébration du mariage ${ }^{7}$.

\section{Contenu}

En ce qui a trait au contenu, on ne peut passer sous silence la grande latitude dont jouissent les stipulants tel qu'il est prévu à l'article 431 C.c.Q., l'équivalent de l'actuel article 463 C.c.Q. : «Il est permis de faire, par contrat de mariage, toutes sortes de stipulations, sous réserve des dispositions impératives de la loi et de l'ordre public».

Malgré cela, le contrat de mariage ne comporte ordinairement que deux éléments principaux : le choix du régime matrimonial et les donations.

\subsection{Le régime matrimonial}

À l'égard des régimes, nous retrouvons dans le nouveau code ceux que nous connaissons déjà, c'est-à-dire la société d'acquêts, qui constitue le régime légal $^{8}$, et la séparation de biens. Leurs règles de fonctionnement respectives demeurent inchangées, exception faite de quelques ajustements concernant la société d'acquêts.

D'abord, le nouvel article 456 C.c.Q. reprend le principe énoncé à l'actuel article 488 C.c.Q. mais en l'élargissant de façon à couvrir non seulement les actions mais toutes les valeurs mobilières. La qualification de propre s'appliquera donc désormais aux valeurs mobilières acquises soit par suite de la déclaration de dividendes sur des valeurs mobilières propres à l'un des époux, soit par suite de l'exercice d'un droit de souscription, de préemption ou d'un autre droit semblable, conféré par des valeurs mobilières propres à l'un des

5. $\quad$ Art. 434 C.c.Q. Pour l'autorisation relative au mariage, voir l'art. 373 C.c.Q.

6. $\quad$ Art. 436 al. 1 C.c.Q.

7. Art. 435 et 436 al. 2 C.c.Q.

8. $\quad$ Art. 432 C.c.Q. 
époux, sauf récompense s'il y a lieu. Question de droit transitoire, l'article $32 \mathrm{de}$ la Loi sur l'application de la réforme du Code civil ${ }^{9}$ prévoit que «la répartition en propres et en acquêts des biens visés à l'article 456 du nouveau code est faite suivant la loi en vigueur lors de leur acquisition».

Ensuite, le nouvel article 476 C.c.Q. vient préciser que «les biens susceptibles de récompense s'estiment d'après leur état au jour de la dissolution du régime et d'après leur valeur au temps de la liquidation». Cette disposition pose toutefois problème. On peut d'une part l'interpréter, à l'instar de Pierre Ciotola et de Nicole Gagnon ${ }^{10}$, comme signifiant de manière implicite que l'ensemble des biens dont il faut tenir compte pour fixer la créance résultant du partage des acquêts doit également être évalué au moment de la liquidation, contrairement à la pratique actuelle qui retient la date où la dissolution produit ses effets $^{11}$. Mais, nous croyons que si le législateur avait voulu modifier cette pratique, il se serait exprimé de façon plus évidente. En outre, nous comprenons mal pourquoi ce dernier adopterait pour l'évaluation des biens de la société d'acquêts, une règle de même nature que celle utilisée dans le cadre du partage de l'indivision communautaire alors que depuis 1989, les acquêts se partagent en valeur.

On peut d'autre part interpréter l'article 476 C.c.Q. de façon plus littérale en en limitant l'application au domaine des récompenses. Par contre, comme le souligne le professeur Jean-Guy Bergeron ${ }^{12}$, l'incohérence persiste puisque les biens doivent alors être évalués à la date de la dissolution pour l'établissement de la valeur des masses et au temps de la liquidation pour le calcul des récompenses, lesquelles se basent sur un enrichissement déterminé à la date de la dissolution.

Deux interprétations donc mais aucune n'est pleinement satisfaisante. Nous favorisons malgré tout la seconde interprétation qui a au moins le mérite de maintenir une certaine concordance entre les dates d'évaluation retenues pour le partage de la société d'acquêts et celles retenues pour le partage du patrimoine familial $^{13}$, une concordance qui se justifie puisque dans les deux cas, il s'agit d'établir une créance ou, en d'autres termes, de partager en valeur. Nous aurions néanmoins préféré qu'aucune modification ne soit apportée à cet aspect du régime matrimonial. À tout événement, l'article 33 L.A.R.C.C. prévoit que

9. L.Q. 1992, c. 57; ci-après appelée «L.A.R.C.C.».

10. Pierre CIOTOLA et Nicole GAGNON, loc. cit., note 3, 49.

11. Il s'agit selon les circonstances de la date du décès, de la demande ou de la cessation de la vie commune selon les articles 465 et 466 C.c.Q.

12. Jean-Guy BERGERON, Le droit patrimonial des conjoints, document pédagogique, 19921993 , pp. 253.

13. Art. 417 C.c.Q. Il est prévu que les biens qui en font partie sont évalués à la date du décès, de la demande ou, sur décision du tribunal, à la date de cessation de la vie commune. 
l'article 476 du nouveau code sera applicable aux sociétés d'acquêts dissoutes avant son entrée en vigueur lorsque l'option sur le partage des acquêts n'a pas encore été exercée par les intéressés et que le délai pour l'exercer n'est pas encore écoulé.

Enfin, comme autres ajustements aux dispositions concernant la société d'acquêts, l'on note des modifications dans l'énoncé de certains articles soit pour des fins de concordance avec le reste du code, c'est le cas notamment aux articles 454 et 455 C.c.Q., soit pour des fins de précision, par exemple à l'article 482 C.c.Q.

\subsection{Les donations}

En plus d'exprimer le choix du régime matrimonial, le contrat de mariage peut comporter différentes donations soit entre vifs, soit à cause de mort ${ }^{14}$. Les principales, et à toutes fins pratiques les seules qui présentent encore un intérêt ${ }^{15}$, sont d'une part, la donation entre vifs de meubles meublants, dont l'utilité se conçoit dans une optique de protection contre les créanciers, et d'autre part, l'institution contractuelle qui, en l'absence de testament, permet d'éviter de recourir à la dévolution légale.

Ces donations font l'objet de règles spécifiques dans le code actuel ${ }^{16}$ et il en sera de même dans le nouveau code mais avec certains changements.

Ainsi, l'article 1840 C.c.Q. modifie le cadre dans lequel une donation par contrat de mariage peut avoir lieu :

Toute personne peut faire une donation entre vifs par contrat de mariage, mais seuls peuvent être donataires les futurs époux, les époux, leurs enfants respectifs et leurs enfants communs nés et à naître, s'ils naissent vivants et viables.

La donation à cause de mort ne peut avoir lieu qu'entre les personnes qui peuvent être bénéficiaires d'une donation entre vifs par contrat de mariage.

Cette disposition, qui n'affecte pas la validité des donations faites selon les règles du code actuel ${ }^{17}$, diffère passablement des articles 818 à 820 C.c.B.C.

\footnotetext{
14. $\quad$ Art. 1839 C.c.Q.

15. Pierre CIOTOLA et Nicole GAGNON, loc. cit., note 3, 54-55; Serge BINETTE, «Régimes matrimoniaux et contrat de mariage», R.D.-Famille-Doctrine-Doc. 2, pp. 59-62.

16. Art. 817-830 C.c.B.C.

17. Pour les donations entre vifs, voir l'article 2 L.A.R.C.C. et pour les donations à cause de mort, voir l'article 105 L.A.R.C.C.
} 
mais au fond, le législateur a simplement voulu mieux refléter la réalité : il a supprimé la possibilité de faire des libéralités par contrat de mariage à des tiers, ce qui devait être plutôt rare, tout en élargissant l'admissibilité des enfants, ce qui tient compte des familles reconstituées, fréquentes de nos jours.

La distinction entre une donation entre vifs et une donation à cause de mort conserve par ailleurs toute son importance. Le nouveau code précise à cet égard que la donation entre vifs implique le désaisissement actuel du donateur et ne porte que sur des biens présents ${ }^{18}$. Cette définition ne fait toutefois que reprendre le droit actuel ${ }^{19}$ de sorte que les techniques de rédaction déjà connues demeurent pertinentes. Soulignons cependant deux changements qui rapprochent en quelque sorte les donations par contrat de mariage des autres donations :

- selon l'article 1822 C.c.Q., la donation entre vifs stipulée révocable suivant la seule discrétion du donateur sera désormais nulle, même si elle est faite par contrat de mariage, à la différence de ce qui est prévu à l'article 824 C.c.B.C.;

- selon l'article 1821 C.c.Q., la donation entre vifs qui impose au donataire l'obligation d'acquitter des dettes ou des charges autres que celles qui existent lors de la donation sera également nulle même si elle est faite par contrat de mariage sauf si la nature de ces autres dettes ou charges est exprimée au contrat et si leur montant y est déterminé, contrairement aux dispositions de l'article 825 C.c.B.C.

Ces nouvelles règles vont affecter les libéralités faites par contrat de mariage avant l'entrée en vigueur du nouveau code. Pour les donations entre vifs valables selon l'article 824 C.c.B.C. mais qui ne le seront plus en raison de l'article 1822 C.c.Q., faute de règle spécifique, on pourrait possiblement appliquer l'article 5 L.A.R.C.C. suivant lequel «les stipulations d'un acte juridique antérieures à la loi nouvelle et qui sont contraires à ses dispositions impératives sont privées d'effet pour l'avenir». Par contre, les donations entre vifs valables selon l'article 825 C.c.B.C. mais qui se heurtent à l'article 1821 C.c.Q. sont maintenues sous réserve de l'article 104 L.A.R.C.C. qui limite à la valeur des biens donnés, l'obligation du donataire d'acquitter des dettes ou des charges à venir dont ni la nature, ni le montant n'ont été déterminés lors du contrat.

Quant à la donation à cause de mort ou institution contractuelle, elle se définit dans le nouveau code comme étant celle où le désaisissement du

18. Art. 1807 et 1818 C.c.Q.

19. Art. 777 et 778 C.c.B.C. 
donateur demeure subordonné à son décès et n'a lieu qu'à ce moment ${ }^{20}$. Là encore, il s'agit de la même notion que dans le droit actuel sauf qu'une modification fondamentale est apportée : en vertu de l'article 1841 C.c.Q., la donation à cause de mort, qu'elle soit à titre particulier ou non, devient en effet révocable à moins de stipulation contraire. On inverse donc complètement la règle prévue à l'article 823 C.c.B.C. mais ce changement aura un impact moins grand qu'il n'y paraît car dans la majorité des cas, l'institution contractuelle est stipulée de façon révocable. Il reste que les parties désirant convenir de l'irrévocabilité devront désormais le dire expressément. Les conséquences d'un tel choix sont précisées au deuxième alinéa de l'article 1841 C.c.Q. :

[...] lorsque le donateur a stipulé l'irrévocabilité de la donation, il ne peut disposer des biens à titre gratuit par acte entre vifs ou par testament, à moins d'avoir obtenu le consentement du donataire et de tous les autres intéressés ou qu'il ne s'agisse de biens de peu de valeur ou de cadeaux d'usage; il demeure, cependant, titulaire des droits sur les biens donnés et libre de les aliéner à titre onéreux.

Pour les institutions contractuelles faites avant l'entrée en vigueur du nouveau code, il est prévu à l'article 106 L.A.R.C.C. que les dispositions de l'article 1841 C.c.Q. leur sont applicables si elles n'ont pas encore été exécutées au jour de l'entrée en vigueur.

Mentionnons finalement que les stipulations d'insaisissabilité seront toujours possibles dans le nouveau droit mais dans un cadre plus restreint, à savoir qu'elles devront être temporaires et justifiées par un intérêt sérieux et légitime selon les termes de l'article 2649 C.c.Q. ${ }^{21}$

\section{Publicité}

Une fois que le contenu du contrat de mariage est arrêté et que l'acte est rédigé, il reste à accomplir les formalités relatives à la publicité.

Ce domaine est sans contredit l'un des plus touchés par la réforme du Code civil sauf qu'en ce qui a trait aux conventions matrimoniales, on remarque plutôt une simplification des règles.

Présentement, on sait que le contrat de mariage fait l'objet d'un avis au registre central des régimes matrimoniaux ${ }^{22}$ et qu'il doit en outre être enregistré

20. $\quad$ Art. 1808 C.c.Q.

21. Sur ce point, voir: Louis PAYETTE, «Des priorités et des hypothèques» dans La Réforme du Code civil, vol. 3, Québec, Les Presses de l'Université Laval, 1993, à la page 31.

22. Art. 474 C.c.Q. (actuel). 
s'il contient des donations $^{23}$. Or, dans la loi nouvelle, la publicité du contrat et des donations s'effectue à un seul registre, celui des droits personnels et réels mobiliers ${ }^{24}$, à moins que le contrat de mariage ne contienne une donation entre vifs d'immeuble, auquel cas, l'inscription au registre foncier devient nécessaire ${ }^{25}$.

Il faut comprendre en effet que le système de publicité prévu au Code civil du Québec s'articule autour de deux registres : le registre foncier, où sont inscrits les droits concernant un immeuble, et le registre des droits personnels et réels mobiliers, où sont inscrits tous les autres droits dont la publicité est requise $^{26}$. Pour un contrat de mariage ne comportant qu'une institution contractuelle ou une donation entre vifs de biens meubles ou les deux, les inscriptions pertinentes au registre des droits personnels et réels mobiliers remplacent donc l'avis au registre central des régimes matrimoniaux de même que l'enregistrement des donations.

Sur la façon de procéder à ces inscriptions, peu de détails sont connus si ce n'est que l'article 2983 C.c.Q. précise qu'elles se font au moyen d'un avis. Il faudra par conséquent attendre le dévoilement de la réglementation gouvernementale pour en savoir plus ${ }^{27}$.

Par contre, il est intéressant de noter que selon l'article 163 L.A.R.C.C. les avis de contrat de mariage déjà inscrits au registre central des régimes matrimoniaux seront portés d'office au registre des droits personnels et réels mobiliers. Les donations déjà enregistrées, quant à elles, conservent leur caractère d'opposabilité sans qu'il y ait besoin d'un renouvellement et peuvent être consultées dans les anciens registres en vertu de l'article 160 L.A.R.C.C.

\section{CONCLUSION}

Bref, il ressort de notre propos que la réforme du Code civil n'apportera pas de bouleversement majeur à la pratique en matière de conventions matrimoniales. La forme des actes, qu'il s'agisse du contrat de mariage ou de la modification d'une convention antérieure, reste la même et les régimes matrimoniaux demeurent tels que nous les connaissons déjà, si ce n'est les ajustements apportés à la société d'acquêts.

\footnotetext{
23. Art. 806 et 807 C.c.B.C.

24. Art. $442,1824,2938$ C.c.Q.

25. La donation est alors inscrite au registre foncier tandis qu'un avis du contrat est inscrit au registre des droits personnels et réels mobiliers.

26. Art. 2970 C.c.Q.

27. Au moment de remettre ce texte, le projet de règlement a été déposé. Voir Projet de règlement sur le registre des droits personnels et réels mobiliers, (1993) 125 G.O. II, 6410.
} 
En fait, outre la simplification des règles de publicité, les changements les plus importants se retrouvent au niveau des donations : premièrement, en ce qui a trait aux personnes pouvant y être parties; deuxièmement, à l'égard des stipulations prohibées en matière de donations entre vifs; et troisièmement, quant à la révocabilité de la donation à cause de mort. Les dispositions qui introduisent ces changements ne semblent cependant pas soulever de difficultés particulières.

L'adaptation à la loi nouvelle dans le domaine des conventions matrimoniales devrait donc, selon nous, se faire sans heurts. Compte tenu des nombreuses réformes qui ont marqué le droit de la famille depuis quelques années, ce répit sera certes bienvenu. 